\title{
EVALUATION OF METHODS TO INCREASE BEAM PULSE WIDTH ON THE DARHT AXIS-II ACCELERATOR*
}

\author{
C. R. Rose ${ }^{\xi}$, C. A. Ekdahl Jr., M. T. Crawford, W. L. Gregory, J. B. Johnson, K. E. Nielsen, M. E. Schulze \\ Los Alamos National Laboratory, PO Box 1663, Mail Stop P912 \\ Los Alamos, NM 87545 USA
}

\section{Abstract}

The second axis (Axis II) of the Dual-Axis Radiographic Hydrodynamic Test (DARHT) facility at Los Alamos National Laboratory (LANL) is an electron linear induction accelerator (LIA) using 74 induction cells, each driven by a separate pulse-forming-networkbased Marx (PFN). The ability to perform precise multipulse radiography is heavily influenced by the temporal beam-energy spread, beam-pulse width, related beam motion, and other focusing and target factors. The nominal beam-pulse flattop width is about $1.6 \mu \mathrm{s}$. A wider beam pulse would allow for increased spacing between kicked pulses and hence more information in radiographic experiments. A flattop pulse-width increase of even 50 to $100 \mathrm{~ns}$ would be of significant value.

In this paper, we present analyses, simulations and data for three low-cost, relatively easy means of lengthening the beam pulse for multipulse radiography. The first involves optimizing the PFN charging voltages such that their respective cell pulse widths are nearly the same. The second method studies the utility of timing the cell voltage pulses such that their trailing edges are aligned instead the leading edges. And the third method, presents experimental data on the effects of increasing the cell core reset currents which increases the available volt-second (V-s) products in the cores yielding increased pulse width.

\section{INTRODUCTION}

The Dual-Axis Radiographic Hydrodynamic Test (DARHT) facility at Los Alamos National Laboratory consists of two linear induction accelerators (LIAs). The Axis-II beam has a pulse width of about $1.6 \mu$ s and energy of $16.5 \mathrm{MeV}[1,2]$. The injector voltage is about 2.1 MV. The Axis-II beam can be chopped into bunches for multipulse radiographic experiments $[3,4,5]$. This work refers specifically to Axis-II.

Figure 1 shows two example beam energy profiles measured at the end of the accelerator. The beam is chopped or kicked at the times labelled P1, P2, P3, and P4. With a $1.6 \mu$ s beam, the spacing between the kicked pulses is about $500 \mathrm{~ns}$.
Note that the last kicked pulse, P4, occurs on a relatively high gradient portion of the energy profile which can lead to increased spot size on the target and reduced radiographic resolution. However, at times it is advantageous to be able to use a slightly higher energy kicked pulse than at other times. Nevertheless, an increase in the usable width of the beam, or the energy profile, would allow for greater flexibility in the timing of the kicked pulses, and for reduced energy gradient at P4, and reduced spot size.

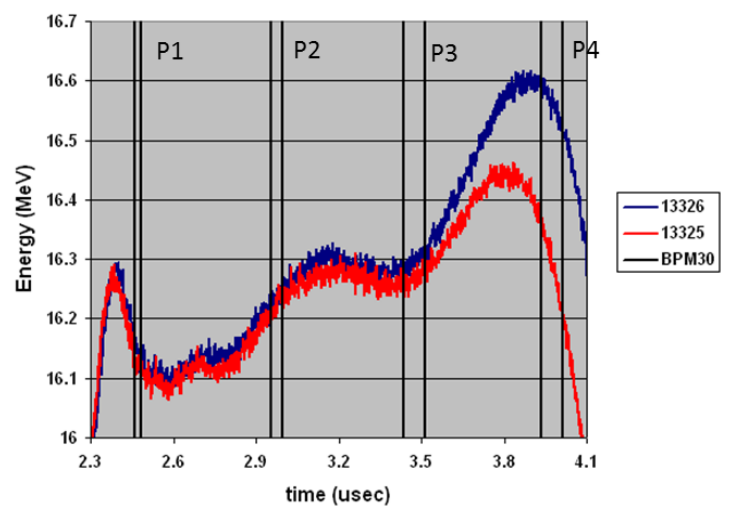

Figure 1. Energy spread over pulse showing four kicked pulses (BPM30) for two shots in which cells 73 and 74 were advanced 550 ns (13325) and with cells 73 and 74 at their nominal time (13326).Timing of kicked pulses noted as P1, P2, P3, and P4.

In this paper, we present a few recently evaluated, relatively easy methods to widen the existing beam pulse without significant changes to the accelerator or infrastructure. The first method is a constrained optimization between total beam energy (sum of all the cell and injector voltages) and maximum cell pulse width. It determines the voltage set point of each of the PFNs that drive the cells such that their respective pulse widths are nearly identical while being subject to a constraint of minimum acceptable total beam energy. Each cell has a unique magnetic-core volt-second (V-s) capability, and properly setting pulse voltages allows for maximum pulse width based on individual core properties. The second method involves retiming the cell pulses such that their trailing edges align instead of their leading edges. The leading edge of the cell pulses are of less importance than

\footnotetext{
${ }^{*}$ Work supported by the U.S. Department of Energy under contract W-7405-ENG-36

₹ email: crose@lanl.gov
} 
the trailing, hence, if the end of the beam pulse can be improved, additional pulse width suitable for radiography may be obtained. The third method focuses on the reset current for the magnetic cores in the cells. We call this reset current though more accurately it could be called core preset current. To achieve sufficient flux swing in the cores, each is preset to a known magnetic flux saturation state prior to each high-voltage pulse. Experiments have shown that the levels of reset current vary by more than $15 \%$ and the timing of the reset pulses can be adjusted to obtain additional improvement in available V-s capability. After combining the available increases in beam-pulse width from these separate methods, we expect to be able to gain over $50 \mathrm{~ns}$ in usable pulse width. We present the results from simulations, experiments and describe measured and expected increases in pulse width due to each of the methods. The paper concludes with a summary of this work and describes future research.

\section{PULSE-WIDTH OPTIMIZATION}

Available, cell-pulse width is inversely proportional to cell voltage which is linked directly to PFN charge voltage. Clearly, reducing the applied voltage to the cells will increase their respective pulse widths in accordance with their V-s products. However, the tradeoff in increasing cell-pulse width is decreased total beam energy. Therefore, the constrained optimization method maximizes cell pulse widths subject to minimumacceptable total-beam energy by finding individual charge voltage settings for each PFN.

Figure 2(a) shows baseline, PFN, charge voltages for shot 19400. Measured cell-voltage pulse widths based on FWHM, are shown in (b). These charge voltages are called "full voltage" settings. The charge voltages are negative, but to simplify notation, the negative sign has been dropped. The measured, average-charge voltage is $82.8 \mathrm{kV}$, and the average pulse width is $2.268 \mu$ s with a standard deviation of $30 \mathrm{~ns}$.
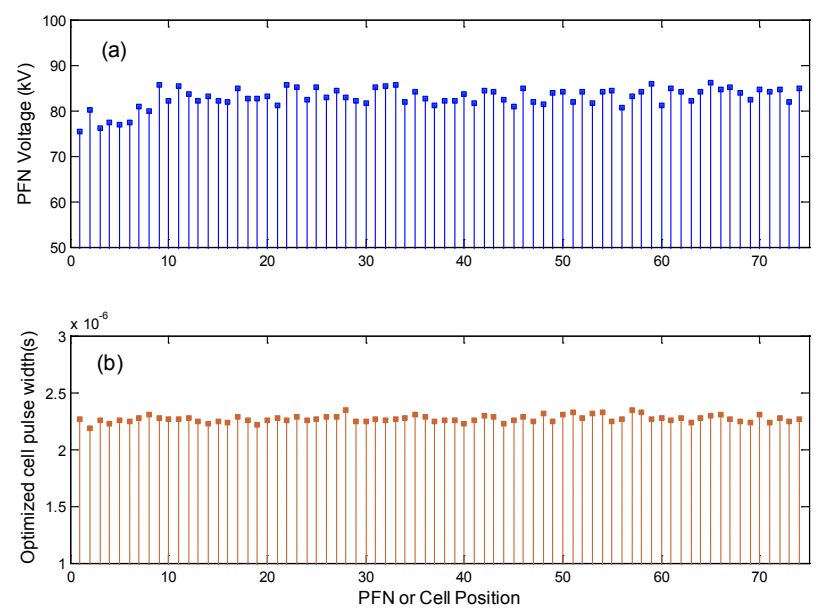

Figure 2. Shot 19400, (a) Baseline PFN charge voltages for each cell, (b) measured pulse width for each cell.
The first six cells are "injector" cells and have a larger beam tube than the other "accelerator" cells. Consequently, they have slightly less magnetic core material and reduced V-s capability [6, 7]. They are operated at a lower voltage than the remainder of the accelerator cells $[8,9,10]$. The measured V-s products for the 74 cells using nominal reset current are shown in Figure 3 . The values range from 440 to $530 \mathrm{mVs}$.

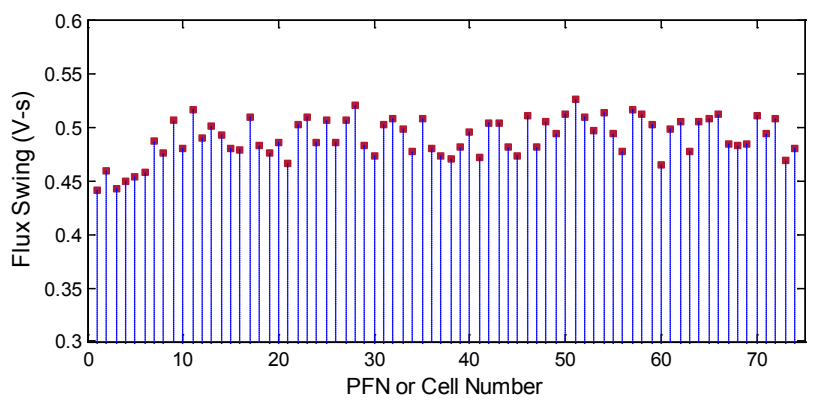

Figure 3. Measured cell V-s capacities with nominal reset current.

The optimization process followed these general steps. First, unloaded cell voltage data were acquired at 74, 76, 78, 80, and 84-kV PFN charge voltage settings. Next, first-order functions were fit to these data such that each cell's pulse width is linearly related to its respective PFN charge voltage. The pulse width as a function of charge voltage, $\tau_{n}$, is given by,

$$
\tau_{n}=f_{n}\left(V_{c, n}\right)
$$

where $n$ represents the number of the PFN or cell, and ranges from 1 to $74, f_{n}$ is the linear function for the $n^{\text {th }}$ cell, and $V_{c, n}$ is the charge voltage for the $n^{\text {th }} \mathrm{PFN}$.

The MATLAB optimizer, fmincon, then searched and adjusted individual PFN charge voltages $V_{c, n}$ in (1), subject to constraints on minimum charge voltage to minimize a cost function. The sum of the charge voltages is directly proportional to total beam energy. The cost function was the standard deviation of the 74 cell pulse widths based on (1) which forced the solution to have nearly identical pulse widths for all cells. The optimizer accounted for the reduced operating voltages of the first six injector cells, and the higher operating voltages of the remaining 68 accelerator cells.

A typical optimizer output is shown in Figure 4 using a constrained, minimum-charge voltage of $74 \mathrm{kV}$. The upper plot (a) shows the resulting PFN charge voltages. Note that the first six are lower voltage than the others because they are injector cells. The lower plot (b) shows the resulting cell pulse widths.

Because of the minimized cost function, the cell-pulse widths are nearly identical with a standard deviation of $1.8 \mathrm{e}-14 \mathrm{~s}$ and mean of $2.304 \mu \mathrm{s}$. Average charge voltage is $81.5 \mathrm{kV}$. 

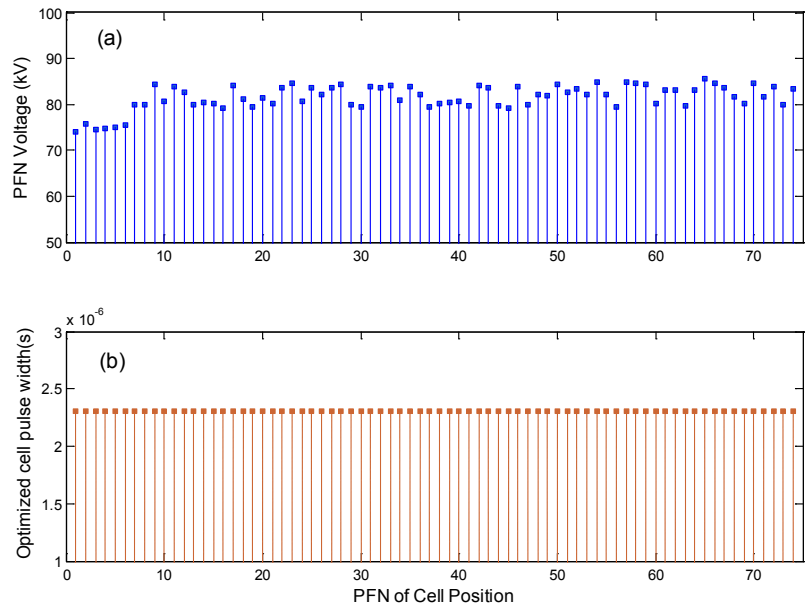

Figure 4. (a) Simulation output showing new cell voltages (PFN charging voltages, minimum of $74 \mathrm{kV}$ ), and (b) resulting cell pulse widths, $2.304 \mu \mathrm{s}$.

We ran several other models using different minimum charge voltage (energy) constraints. These results are shown in Figure 5. For comparison, the present baseline average pulse width, $2.268 \mu \mathrm{s}$, and average charge voltage, $82.8 \mathrm{kV}$, values are shown in the upper left corner. The values from the constrained optimizations are paired by average pulse width in seconds, and average charge voltage in $\mathrm{kV}$.

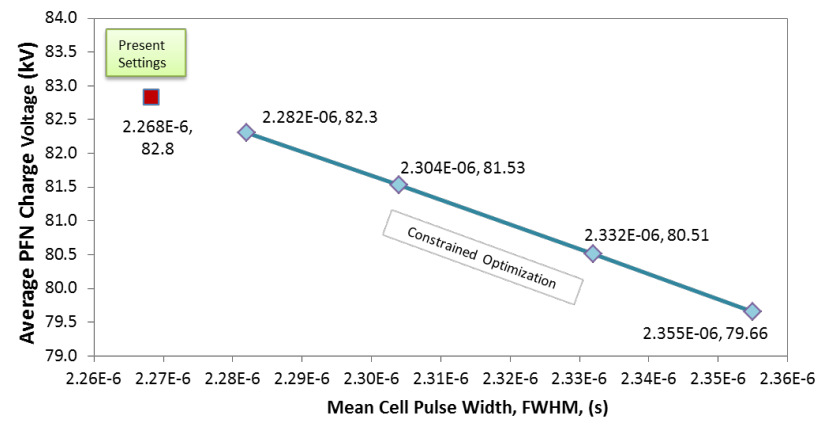

Figure 5. Plot showing multiple optimizer outputs and relationship of pulse width to average charging voltage.

The average pulse widths increase from the baseline average pulse width by $14,36,64$ and $87 \mathrm{~ns}$, and the average charge voltage decreases by $0.6,1.5,2.8$ and 3.8 percent. Again, these are approximate reductions in total beam energy, corresponding to increased available pulse width.

\section{TRAILING-EDGE ALIGNMENT}

Normally, the cell voltages are timed to be coincident at their leading edges. Noticing that the largest energy profile excursion occurs near the trailing edge of the beam energy profile (see Figure 1), we evaluated the feasibility of retiming the cell voltages to have their respective trailing edges aligned. We examined three cases: nominal timing for a baseline; group alignment; and individual timing of trailing edges. The cells on Axis-II are timed in groups of four using four-channel Trigger Chassis except for the last six cells which are timed in one group of three, and the last three which are timed and triggered individually. Also, these analyses use unloaded cell voltages, and are approximations as to the timing and overall shape that would be expected with loaded cell waveforms. The injector voltage data is not included in these analyses.

Figure 6 shows 74 cell voltage waveforms at the baseline, or nominal timing. These are intended to have identical timing at the leading edge; however, because of timing variation within each trigger group, there is noticeable spread at the leading edge. Figure 7 shows the computed baseline energy profile using an analysis window from 2.45 to $4.05 \mu$ s. The energy profiles (sum of cell voltages) for group and individual timing are shown on the same figure for easy comparison. Energy profiles are used to compare relative increases in usable pulse width.

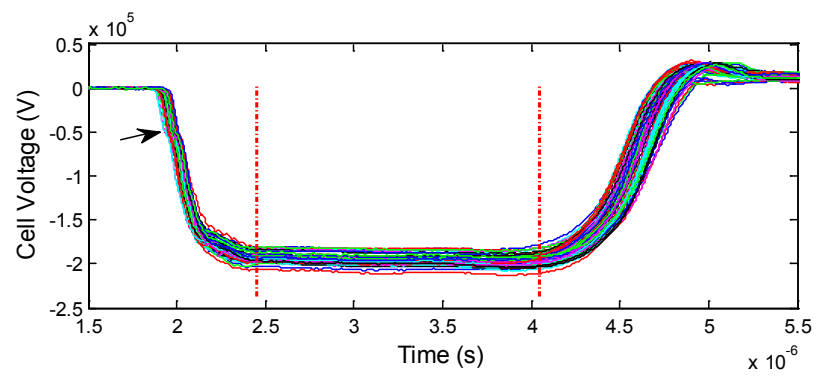

Figure 6. Unloaded cell voltage waveforms (74) with nominal timing. Window markers are at 2.45 and $4.05 \mu \mathrm{s}$.

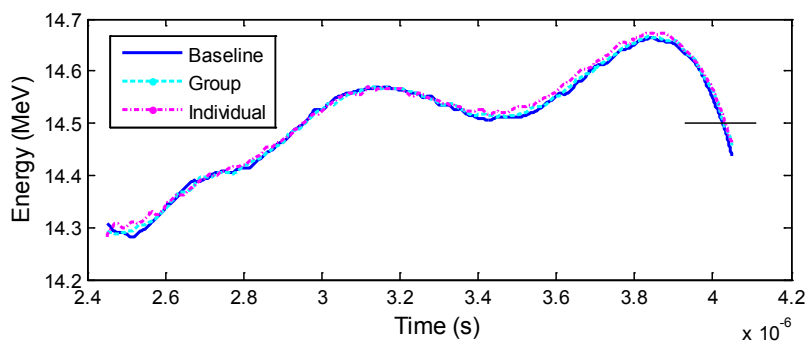

Figure 7. Computed energy profiles for baseline, group, and individual trailing-edge timing. Timing window is from 2.45 to $4.05 \mu \mathrm{s}$. The Line shows location of pulse-increase measurements.

In the second case, we simulated the timing of the trailing edges by trigger group. The FWHM method was used to minimize effects from amplitude instrumentation errors. The timing of the 21 groups of cells was adjusted to match their half-maximum levels at the trailing edges. Figure 8 shows these results. Again, because of timing variation within trigger groups, there is noticeable timing spread even at the trailing edges.

The computed energy profile for group-based timing is shown in Figure 7. 


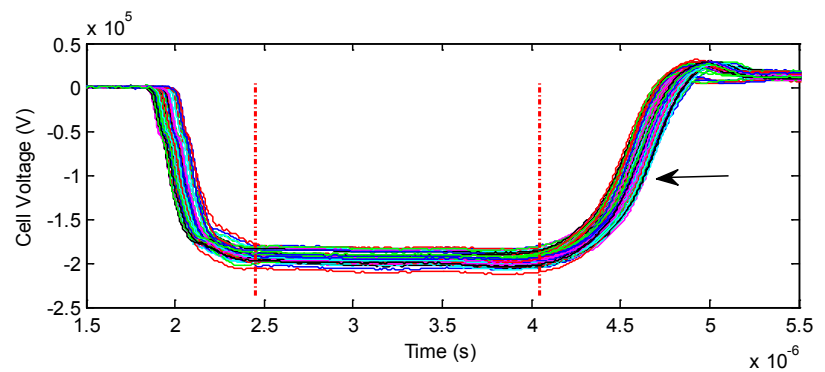

Figure 8 . Unloaded cell voltage waveforms with trailing edge trigger group timing. Markers are at 2.45 and $4.05 \mu \mathrm{s}$.

The third timing alignment method simulated the possible improvement in energy profile and increased, beam pulse width assuming that each of the 74 cells can be individually timed at their trailing edges. We should mention that at present, the DARHT facility is not equipped to do this. Figure 9 shows the results from these simulations. Because in this simulation, it is assumed that the cells can be timed individually, the correlation of trailing edge timing is very good as indicated by the arrow.

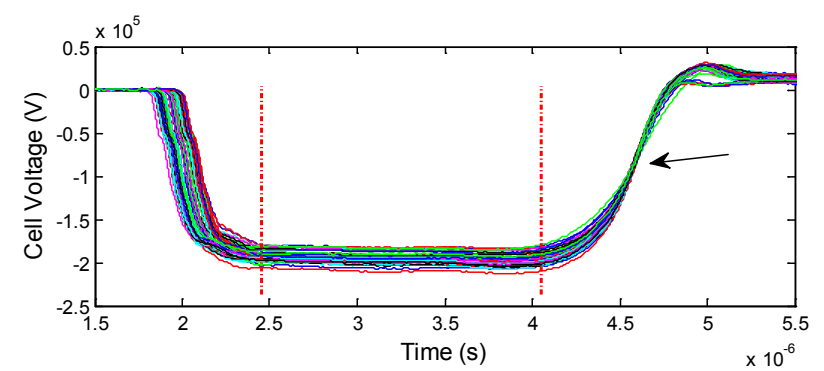

Figure 9. Unloaded cell voltage waveforms with trailing edge individual timing. Note the alignment at the arrow marker. Window markers are at 2.45 and $4.05 \mu \mathrm{s}$.

The analysis window is from 2.45 to $4.05 \mu \mathrm{s}$, and the computed energy profile is also shown in Figure 7 . Note that the three profiles closely overlay with minor variations.

Figure 10 shows the energy profiles of Figure 7 using an expanded scale at the trailing edge. Assuming an energy level of $14.5 \mathrm{MeV}$, the increase in width from the baseline to the trailing-edge-aligned group is about $3 \mathrm{~ns}$, and the increase in width from the baseline to the individually timed profile is about 6 ns. Both of these time increases are within the timing error and jitter of the system and consequently of limited utility.

There has been other work done to adjust the timing of groups of cells to improve the energy profile, reduce its spread, and lengthen the usable beam pulse [11]. The work described in this section analyzed a much simpler method of adjusting the timing of the trailing edges to obtain additional pulse width. Work is continuing to adapt and utilize loaded-cell models which are expected to yield more practical results.

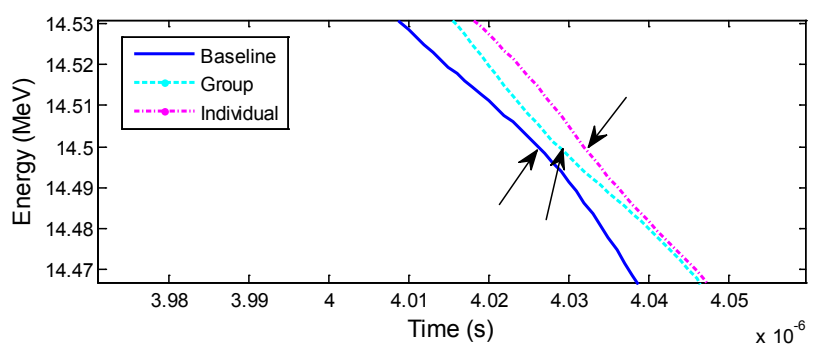

Figure 10. Graphic showing the baseline, group timed, and individually timed energy profiles.

\section{CORE RESET CURRENT}

In DARHT Axis-II, the Metglas cores in the 74 induction cells are reset for maximum magnetic flux swing prior to each high-voltage pulse by 19 Reset Chassis. All of the Reset Chassis drive four cells except for the last unit which drives two. The first 18 (cells 1-72) are normally charged to $7 \mathrm{kV}$, and the last unit because it only drives two cells (cells 73 and 74), is normally charged to $5.5 \mathrm{kV}$. Reset current was originally intended to be $600 \mathrm{~A}$ [7].

Two shots were executed to measure the influence of core reset levels on available cell-pulse width. The first, 19260, used nominal Reset Chassis charge voltages with concomitant levels of reset currents. Figure 11 shows waveforms for the 20, instrumented, reset-current channels used in this analysis. The spikes at $t=0 \mu$ s are the PFNs firing in which they draw additional current from their respective Reset Chassis. Note that they fire after the reset-current peak for most of these systems.

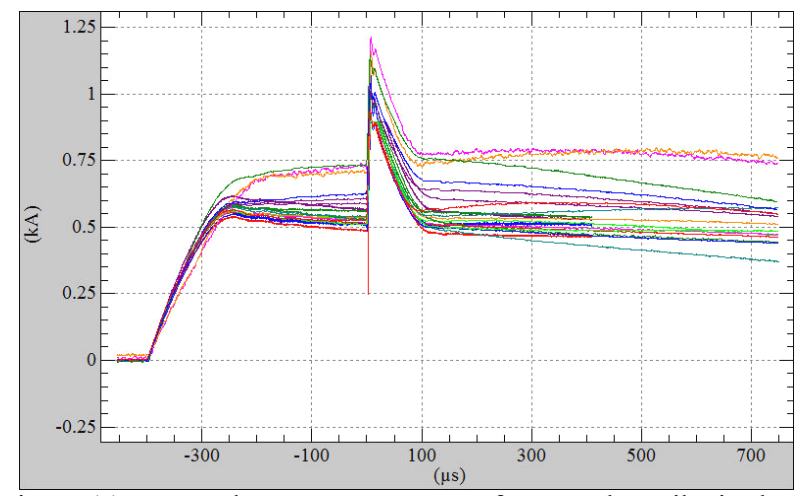

Figure 11. Example reset current waveforms. The spike in the middle is when the PFNs erect.

In the second shot, 19261, the charge voltages to each of the 19 Reset Chassis were increased by $1 \mathrm{kV}$ (cells 1 72 at $8 \mathrm{kV}$ charge, and cells 73 and 74 at $6.5 \mathrm{kV}$ charge). As expected, the V-s capabilities increased. In both shots, the first eight PFNs were charged to $-78 \mathrm{kV}$, and the rest were charged to $-82 \mathrm{kV}$. 
Figure 12 shows measured increases in both cell-pulse width and core reset current. The average increase in pulse width for the 20 systems is $29 \mathrm{~ns}$.

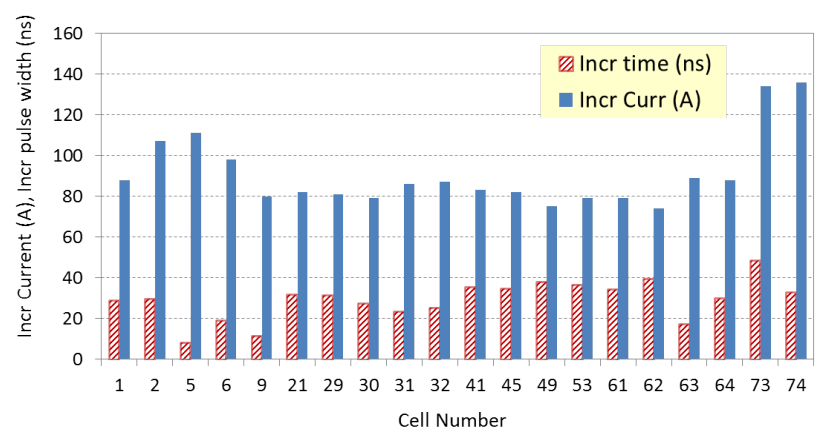

Figure 12. Measured increase in cell pulse width with increased core reset current for the 20 measured cells. Shot 19260 baseline and 19261 with increased reset currents.

The pulse widths were measured as close as possible to the flattop portion of the waveforms while trying to maintain relatively clean and sharp measurement edges. Thus, for cells 1-6, the pulse width measurement was done at $-190 \mathrm{kV}$, and for the remaining cells, it was done at $-195 \mathrm{kV}$. Other pulse-width measurement levels may yield slightly different results.

After observing the performance of 20 systems, we next examined the behavior of all 74 cells and how increased reset current might impact total beam energy.

Figure 13 shows the 74 measured, cell-voltage waveforms for shot 19260 . The cell-voltage waveforms from shot 19261 are very similar and are not shown.

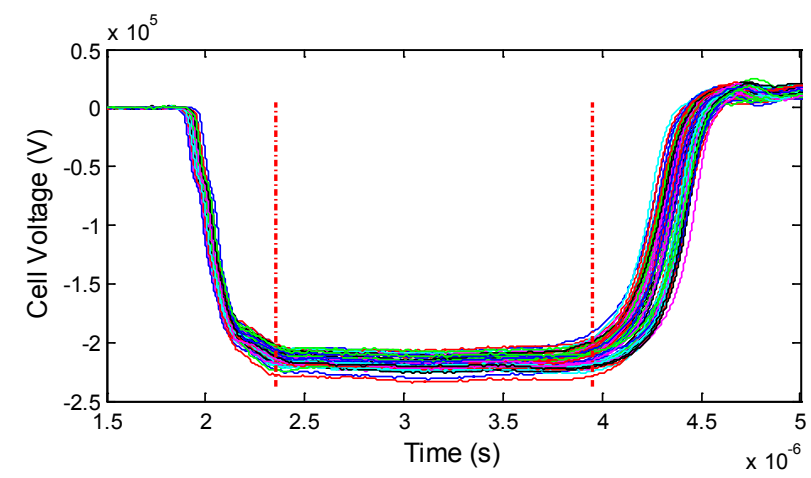

Figure 13. Cell voltage waveforms (74) from shot 19260. Energy profile is measured from 2.35 to $3.95 \mu \mathrm{s}$. Waveforms from 19261 are very similar.

The markers indicate the window start and stop times at 2.35 to $3.95 \mu$ s. The measured energy profiles, for the unloaded cells, are shown in Figure 14 for both shots. For shot 19260 , the peak energy is $16.22 \mathrm{MeV}$, and for shot 19261 , the peak energy is $16.14 \mathrm{MeV}$. These numbers show a small reduction in the energy profile (sum of all the cell voltages) of about 0.5 percent. This reduction is related to the increased Reset Chassis charge voltage settings, i.e., 7 to $8 \mathrm{kV}$, and 5.5 to $6.5 \mathrm{kV}$ in our experiments, that appear as incrementally larger bias voltages on the cells.

In practice, we expect that the amount of beam energy, in terms of percentage reduction, will be smaller than this amount because the 2.1-MV injector voltage and the effects of beam loading need to be taken into account.

Examining the trailing edges of the waveforms in Figure 14, and using a somewhat arbitrary energy level of 15.8 MeV (MV), the increase in available beam pulse is about $26 \mathrm{~ns}$.

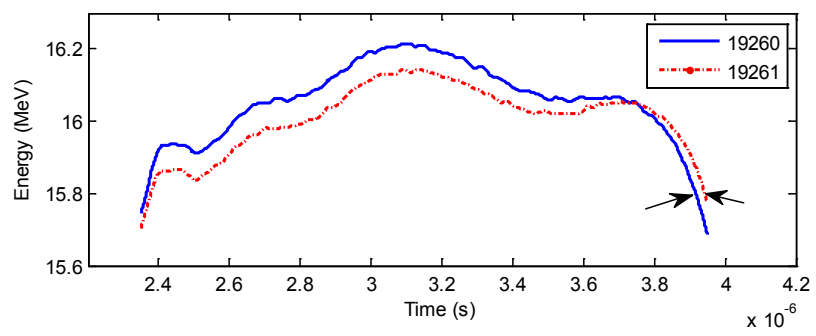

Figure 14. Overlay of the energy profiles from shots 19260 and 19261 using the analysis window from 2.35 to $3.95 \mu$ s. All 74 cells are used to create these profiles. The arrows indicate a possible 26-ns increase in available pulse width.

Figure 11 shows that most of the PFNs are fired after the peaks of their respective core reset current pulses. By simply retiming the Reset Chassis (while keeping the charging voltages at nominal 5.5 and $7-\mathrm{kV}$ values) such that the PFNs are fired at or near the peaks, we expect that the average available beam pulse and energy profile could be increased by $13 \mathrm{~ns}$. This number is based on a cell pulse width per core reset sensitivity of $0.33 \mathrm{~ns} / \mathrm{A}$.

\section{SUMMARY}

This paper described the results from three methods we studied to increase the usable, beam-pulse width of the DARHT Axis-II accelerator without significant changes to the accelerator or infrastructure. The first method reprogrammed the PFN charge voltages for optimal cell pulse width. Simulations show that usable pulse width could be increased over $60 \mathrm{~ns}$ depending on allowable total beam energy reduction.

The second method examined the feasibility of retiming the cell voltages to be coincident at their trailing edges. Simulations of unloaded cells show a marginal improvement of a few nanoseconds. Work is continuing to adapt and use loaded cell models which will yield a more realistic analysis.

In the third method, we showed measured, cell-pulsewidth increases due to increased reset currents. The data show that by increasing the reset current, the usable pulse widths could be increased by up to $26 \mathrm{~ns}$. Other levels of reset current will yield different pulse-width values. We noted that the Reset Chassis are not presently timed to have their peak output currents coincide with the firing of the PFNs, and that by simply retiming them for maximum 
current, up to 13-ns of usable pulse width could be obtained at present Reset Chassis charge voltage settings.

We expect that a combination of optimized PFN charge voltages, possibly retiming the trailing edges, and increasing the reset currents will yield at least $50 \mathrm{~ns}$ of increased beam and energy-profile pulse width.

\section{ACKNOWLEDGEMENTS}

The authors gratefully acknowledge D. Moir, T. McCuistian, J. Barraza, the DARHT Axis-II Operators and management for their support of this research.

\section{REFERENCES}

[1] S. Nath, "Linear Induction Accelerators at the Los Alamos National Laboratory DARHT Facility," Proc. Linear Accelerator Conference, LINAC 2010, Vol.1, 750, (2010).

[2] W. L. Waldron, and L. L. Reginato, "Design and Performance of the DARHT Second Axis Induction Cells and Drivers," Twenty-Fourth International Power Modulator Symposium, pp. 179-182, 26-29 June 2000

[3] M. J. Burns, B.E. Carlsten, T.J.T. Kwan, D.C. Moir, D.S. Prono, S.A. Watson, E.L. Burgess, H.L. Rutkowski, G.J. Caporaso, Y.-J. Chen, Y-J. (Judy) Chen, S. Sampayan, and G. Westenskow, "DARHT Accelerators Update and Plans for Initial Operation," Proc. 1999 Particle Accelerator Conference, New York, NY

[4] M. Schulze, E. O. Abeyta, P. Aragon, R. Archuleta, J. Barraza, D. Dalmas, C. Ekdahl, K. Esquibel, S. Eversole, R. Gallegos, J. Harrison, J. Johnson, E. Jacquez, P. Marroquin, B. Trent McCuistian, R. Mitchell, N. Montoya, S. Nath, L. Rowton, R. Scarpetti, M. Schauer, R. Anaya, G Caporaso, F. Chambers, Y.J. Chen, S. Falabella, G. Guethlein, J. McCarrick, B. Raymond, R. Richardson, J. Watson, J. Weir, H. Bender, W. Broste, C. Carlson, D. Frayer, D. Johnson, A. Tipton, and C. Y. Tom, T. C. Genoni, T. P. Hughes, C. Thoma, "Commissioning the DARHT-II Scaled Accelerator Downstream Transport," Particle Accelerator Conference, 2007, pp. 2627-2629, 25-29 June 2007.

[5] E. G. Cook, B. S. Lee, S. A. Hawkins, E. M. Anaya, F. V. Allen, B. C. Hickman, J. S. Sullivan, and C. A. Brooksby, "Solid-State Kicker Pulser for DARHT-2," Pulsed Power Plasma Science, Vol. 1, pp. 632-635 (2001).

[6] E. A. Rose, D. A. Dalmas, J. N. Downing, R.D. Temple, "Testing Metglas for use in DARHT Accelerator Cells," Pulsed Power Plasma Science, vol.2, pp. 17041706, 17-22 June 2001

[7] E. A. Rose, D.A. Dalmas, J.N. Downing, R.D. Temple, "Testing pulse forming networks with DARHT accelerator cells," Pulsed Power Plasma Science, vol. 2, pp. 1700-1703, 17-22 June 2001.

[8] T. P. Hughes, T. C. Genoni, H. A. Davis, M. Kang, and B. A. Prichard, "Numerical Model of the DARHT Accelerating Cell," Pulsed Power Conference, p. 143 (2005)

[9] B. A. Prichard, J. Barraza, M. Kang, K. Nielsen, F. Bieniosek, K. Chow, W. Fawley, E. Henestroza, L. Reginato, W. Waldron, R. Briggs, T. Hughes, T. Genoni, "Technological Improvements in the DARHT II Accelerator Cells," Proc 2005 Particle Accelerator Conference, pp. 169-173 (2005).

[10] J. Barraza, T. Ilg, K. Nielsen, M. Kang, V. Smith, K. Chow, C. Peters, D. Munson, B. Prichard, "Mechanical Engineering Upgrades to the DARHT-II Induction Cells," Pulsed Power Conference, 2005 IEEE, pp. 402-406, 1317 June 2005.

[11] C. R. Rose, C. Ekdahl, and M. Schulze, "Beamenergy-spread minimization using cell-timing optimization," Phys Rev Spec Topics Accel and Beams, 15, 040403 (2012) 